\title{
Comparison between four dissimilar solar panel configurations
}

\author{
K. Suleiman ${ }^{1}$ U. A. Ali ${ }^{1,2} \cdot$ Ibrahim Yusuf ${ }^{3}$ A. D. Koko ${ }^{2} \cdot$ S. I. Bala ${ }^{3}$
}

Received: 19 December 2014/ Accepted: 11 March 2017/Published online: 22 March 2017

(c) The Author(s) 2017. This article is an open access publication

\begin{abstract}
Several studies on photovoltaic systems focused on how it operates and energy required in operating it. Little attention is paid on its configurations, modeling of mean time to system failure, availability, cost benefit and comparisons of parallel and series-parallel designs. In this research work, four system configurations were studied. Configuration I consists of two sub-components arranged in parallel with $24 \mathrm{~V}$ each, configuration II consists of four sub-components arranged logically in parallel with $12 \mathrm{~V}$ each, configuration III consists of four sub-components arranged in series-parallel with $8 \mathrm{~V}$ each, and configuration IV has six sub-components with $6 \mathrm{~V}$ each arranged in series-parallel. Comparative analysis was made using Chapman Kolmogorov's method. The derivation for explicit expression of mean time to system failure, steady
\end{abstract}

K. Suleiman

kabiru.suleman@yahoo.ca

U. A. Ali

ubahamad@yahoo.co.uk

Ibrahim Yusuf

iyusuf.mth@buk.edu.ng

A. D. Koko

adanbabako@yahoo.co.uk

S. I. Bala

saminub@yahoo.com

1 Department of Mathematics, Federal University, Dutse, Nigeria

2 Department of Mathematics, Usmanu Danfodiyo University, Sokoto, Nigeria

3 Department of Mathematical Sciences, Bayero University Kano, Kano, Nigeria state availability and cost benefit analysis were performed, based on the comparison. Ranking method was used to determine the optimal configuration of the systems. The results of analytical and numerical solutions of system availability and mean time to system failure were determined and it was found that configuration I is the optimal configuration.

Keywords Reliability · Solar panel · Availability . Photovoltaic

\section{Introduction}

In the literature of reliability comparative analysis, most of the studies focus on two unit standby systems. Little attention is paid on reliability comparison between systems configured as series-parallel. Reliability analyses of various systems design have been explored by Garg et al. (2010), Srinivasa and Naikan (2014), Yazdanpanah (2014) and Yusuf (2014). Sarhan et al. (2004) dealt with reliability equivalence of series-parallel system, Sarhan (2009) studied the analysis of reliability equivalence factor of general series-parallel system, Cichocki (2001) studied limit reliability of some homogeneous regular series-parallel and parallel-series systems, Juang et al. (2008) have evaluated a knowledge management system for seriesparallel availability optimization and design, Moghaddam et al. (2008) discussed on the reliability optimization of series-parallel systems with a choice of redundancy strategies using genetic algorithm, Sun et al. (2008) studied the reliability modeling and analysis of serial-parallel hybrid multi operational manufacturing system by considering dimensional quality. Levetin and Lisnianski (1999) have studied joint redundancy and maintenance 
optimization for multistate series-parallel systems. Yalaoui et al. (2005) have evaluated the reliability allocation problem in a series-parallel system. Kolowrocki (1994) evaluated Limit reliability functions of some series-parallel and parallel-series systems. Moustafa (1998) discussed Reliability model of series-parallel systems. According to Deepankar et al. (2010) reported that the power produced by the photovoltaic (PV) system is used to operate the required heating/cooling equipment inside the greenhouse. A greenhouse may be defined as a sophisticated structure, providing ideal conditions for satisfactory plant growth and production throughout the year. To maintain favorable conditions in the greenhouse during off/pre and post harvesting some additional sources are required. The solar PV system is one of the energy sources, which work at the lowest cost. The various components of the PV system are solar panel, logic based charge controller, battery bank and converter DC/AC.

The problem considered in this paper is different from the work of the discussed authors above. Most of the published articles on solar (photovoltaic) system focus on how it operates, maximum energy required to operate it. Little or no attention is paid to different type of configuration design. In this study, we design four different configuration of PV solar panel with maximum voltage of 24 each, using parallel and series-parallel design in the study we derive the explicit expression of mean time to system failure and availability, a MATLAB software is used to obtain numerical results obtained and MAPLE software is used to interpret the analytic results obtained the two results revealed that configuration $I$ is the optimal configuration. In this paper, we construct four distinct redundant photovoltaic systems and derived their corresponding mathematical models. Furthermore, we study reliability characteristics of each model using Kolmogorov's forward equation method. The focus of our analysis is primarily to capture the effect of both failure and repair rates on the measures of system effectiveness like mean time to system failure (MTSF), availability and profit, to compare the four configurations base on assumed numerical values to determine the optimal configuration and to rank the four configurations using analytical results. We also looked at the effect of the system design.

The organization of the paper is as follows. "Notations, assumptions and system description" present notations, assumptions and the description of the system. "Models formulations" present formulations of the models. "Analytical comparison of four configurations" present analytical comparisons between configurations. The results of our numerical comparisons between configurations are presented in "Numericals comparison of four configurations". Finally, we make a concluding remark in "Conclusion".
Notations, assumptions and system description

\section{Assumptions}

Systems have redundant standby units.

Repair is immediate.

Switching from standby to operation is perfect.

All the four systems have the same failure and repair rates.

\section{Notations and nomenclature}

$\alpha$ : Unit failure rate

$\beta$ : Unit repair rate

$\mathrm{Av}_{i} ; i=1,2,3,4$, Availability of system

$\mathrm{MTSF}_{i} i=1,2,3,4$, Mean time to system failure of system

$P(t)=$ Probability row vector

$E[\cdot]=$ Relation used to compute the expected time

to reach an absorbing state

In this paper four configurations of series parallel solar panels are considered with an aim to increase their efficiency. The description of the four system configurations are as follows: system (configuration) I has two sub-components arranged logically in parallel with $24 \mathrm{~V}$ each, failure of any sub-component does not cause the complete failure of the system. System II has four sub-components arranged in parallel with $12 \mathrm{~V}$ each with two alternative paths. The system can only fail when two sub-components failures occur simultaneously within the two paths. System III has four sub-components with two sub-components $\mathrm{B}_{1}$ and $\mathrm{B}_{2}$ arranged logically in parallel to sub-component $\mathrm{A}$ and $\mathrm{C}$ with $8 \mathrm{~V}$ each. A failure of the system is only said to have occur if $\mathrm{A}$ or $\mathrm{C}$ failed or simultaneous failure of $B_{1}$ and $B_{2}$. System IV has six sub-components with $\mathrm{B}_{1}$ and $\mathrm{B}_{2}$ arranged in parallel to A,C,D and E. System failure occurs if any of $\mathrm{A}, \mathrm{C}, \mathrm{D}$ and $\mathrm{E}$ failed or simultaneous failure of $B_{1}$ and $B_{2}$.

\section{Models formulations}

\section{Availability and meantime to system failure of configuration $I$}

According to Wang et al. (2006), let $P(t)$ be the probability that at time $t$ there are $n$ components working in the system. 
Then the initial conditions for this problem are stated as follows:

$P(0)=\left[P_{0}(0), P_{1}(0), P_{2}(0), P_{3}(0)\right]=[1,0,0,0]$

From Fig. 1 we obtain the following first order linear differential equation.

$P_{0}^{\prime}(t)=-2 \alpha P_{0}(t)+\beta P_{1}(t)+\beta P_{2}(t)$

$P_{1}^{\prime}(t)=-(\alpha+\beta) P_{1}(t)+\alpha P_{0}(t)+\beta P_{3}(t)$

$P_{2}^{\prime}(t)=-(\alpha+\beta) P_{2}(t)+\alpha P_{0}(t)+\beta P_{3}(t)$

$P_{3}^{\prime}(t)=-2 \beta P_{3}(t)+\alpha P_{1}(t)+\alpha P_{2}(t)$

Equation (3.1) above can be written in the form

$\dot{P}=X_{1} P$,

where $X_{1}=\left[\begin{array}{cccc}-2 \alpha & \beta & \beta & 0 \\ \alpha & -(\alpha+\beta) & 0 & \beta \\ \alpha & 0 & -(\alpha+\beta) & \beta \\ 0 & \alpha & \alpha & -2 \beta\end{array}\right]$

Equation (3.2) above can be written in the matrix form as:

$$
\left(\begin{array}{c}
\dot{P}_{0} \\
\dot{P}_{1} \\
\dot{P}_{2} \\
\dot{P}_{3}
\end{array}\right)=\left(\begin{array}{cccc}
-2 \alpha & \beta & \beta & 0 \\
\alpha & -(\alpha+\beta) & 0 & \beta \\
\alpha & 0 & -(\alpha+\beta) & \beta \\
0 & \alpha & \alpha & -2 \beta
\end{array}\right)
$$

In the steady state all the derivative equal to zero, thus from (3.2) above we have

$X_{1} P(\infty)=0$

Thus, Eq. (3.3) above can be written in matrix form as

$$
\left(\begin{array}{cccc}
-2 \alpha & \beta & \beta & 0 \\
\alpha & -(\alpha+\beta) & 0 & \beta \\
\alpha & 0 & -(\alpha+\beta) & \beta \\
0 & \alpha & \alpha & -2 \beta
\end{array}\right)\left(\begin{array}{l}
P_{0}(\infty) \\
P_{1}(\infty) \\
P_{2}(\infty) \\
P_{3}(\infty)
\end{array}\right)=\left(\begin{array}{l}
0 \\
0 \\
0 \\
0
\end{array}\right)
$$

Using the normalizing condition below, it follows that

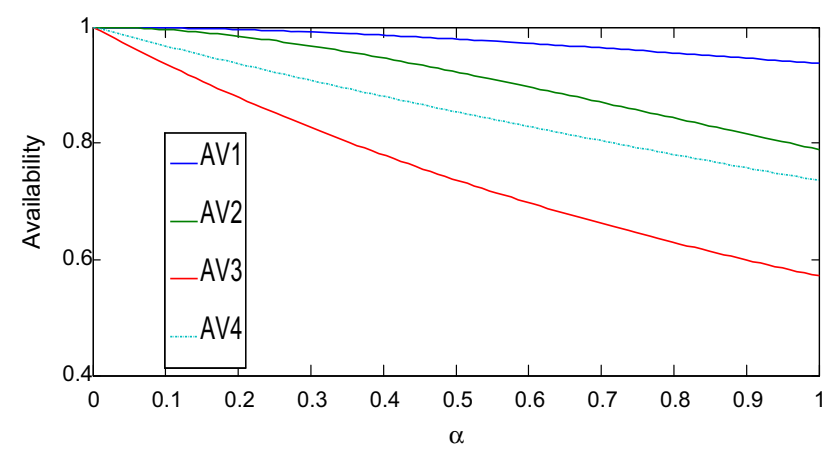

Fig. 1 Availability versus $\alpha$ $\sum_{i=0}^{3} P_{i}(\infty)=1$

Following Wang et al. (2006), we substituted Eq. (3.5) in the last row of (3.4) to obtain

$$
\left(\begin{array}{cccc}
-2 \alpha & \beta & \beta & 0 \\
\alpha & -(\alpha+\beta) & 0 & \beta \\
\alpha & 0 & -(\alpha+\beta) & \beta \\
1 & 1 & 1 & 1
\end{array}\right)\left(\begin{array}{l}
P_{0}(\infty) \\
P_{1}(\infty) \\
P_{2}(\infty) \\
P_{3}(\infty)
\end{array}\right)=\left(\begin{array}{l}
0 \\
0 \\
0 \\
1
\end{array}\right)
$$

Solving Eq. (3.6) to obtain $P_{0}(\infty), P_{1}(\infty) P_{2}(\infty)$, $P_{3}(\infty)$ we therefore, obtained the explicit expression for steady state availability as follows:

$\operatorname{Av}_{1}(\infty)=P_{0}(\infty)+P_{1}(\infty)+P_{2}(\infty)=\frac{\beta^{2}+2 \alpha \beta}{\alpha^{2}+2 \alpha \beta+\beta^{2}}$

Now to evaluate the MTSF for configuration I, following Wang and Kuo (2000) and Wang et al. (2006), the MTSF of a system could be obtained by deleting the rows and column of the absorbing (failure) state and transposing the new matrix $H_{1}$ as given in Eq. (3.8). The expected time to reach an absorbing state is

$E\left[T_{p(0) \rightarrow p(\text { absorbing })}\right]=P(0)\left(-H_{1}^{-1}\right)\left[\begin{array}{l}1 \\ 1 \\ 1\end{array}\right]$,

where $H_{1}=\left(\begin{array}{ccc}-2 \alpha & \beta & {[1} \\ \alpha & -(\alpha+\beta) & 0 \\ \alpha & 0 & -(\alpha+\beta)\end{array}\right)$. we have $E\left[T_{p(0) \rightarrow p \text { (absorbing) }}\right]=\operatorname{MTSF}_{1}=\frac{3 \alpha+\beta}{2 \alpha^{2}}$.

\section{Availability and meantime to system failure of configuration II}

For the analysis of availability case of configuration II, we define $P_{i}(t), i=1,2,3, \ldots, 6$ to be the probability that the system at time $t \geq 0$ is in state $S_{i}$. Also let $P(t)$ be the probability row vector at time $t \geq 0$. The initial condition for this problem is: $P(0)=\left[P_{0}(0), P_{1}(0), P_{2}(0), P_{3}(0)\right.$, $\left.P_{4}(0), P_{5}(0), P_{6}(0)\right]=[1,0,0,0,0,0,0]$.

We obtain the following differential equations

$P_{0}^{\prime}(t)=-2 \alpha P_{0}(t)+\beta P_{1}(t)+\beta P_{2}(t)$

$P_{1}^{\prime}(t)=-(2 \alpha+\beta) P_{1}(t)+\alpha P_{0}(t)+\beta P_{3}(t)+\beta P_{4}(t)$

$P_{2}^{\prime}(t)=-(2 \alpha+\beta) P_{2}(t)+\alpha P_{0}(t)+\beta P_{5}(t)+\beta P_{6}(t)$

$P_{3}^{\prime}(t)=-\beta P_{3}(t)+\alpha P_{1}(t)$

$P_{4}^{\prime}=-\beta P_{4}(t)+\alpha P_{1}(t)$

$P_{5}^{\prime}=-\beta P_{5}(t)+\alpha P_{2}(t)$

$P_{6}^{\prime}(t)=-\beta P_{6}(t)+\alpha P_{2}(t)$

With initial conditions $P(0)=\left[P_{0}(0), P_{1}(0), P_{2}(0)\right.$, $\left.P_{3}(0), P_{4}(0), P_{5}(0), P_{6}(0)\right]=[1,0,0,0,0,0,0]$. 
Equation (3.9) could be written in the form of matrix as: $\dot{P}=X_{2} P$,

which is in matrix form as:

$$
\begin{aligned}
{\left[\begin{array}{l}
P_{0}^{\prime}(t) \\
P_{1}^{\prime}(t) \\
P_{2}^{\prime}(t) \\
P_{3}^{\prime}(t) \\
P_{4}^{\prime}(t) \\
P_{5}^{\prime}(t) \\
P_{6}^{\prime}(t)
\end{array}\right]=} & {\left[\begin{array}{ccccccc}
-2 \alpha & \beta & \beta & 0 & 0 & 0 & 0 \\
\alpha & -(2 \alpha+\beta) & 0 & \beta & \beta & 0 & 0 \\
\alpha & 0 & -(2 \alpha+\beta) & 0 & 0 & \beta & \beta \\
0 & \alpha & 0 & -\beta & 0 & 0 & 0 \\
0 & \alpha & 0 & 0 & -\beta & 0 & 0 \\
0 & 0 & \alpha & 0 & 0 & -\beta & 0 \\
0 & 0 & \alpha & 0 & 0 & 0 & -\beta
\end{array}\right] } \\
& {\left[\begin{array}{l}
P_{0}(t) \\
P_{1}(t) \\
P_{2}(t) \\
P_{3}(t) \\
P_{4}(t) \\
P_{5}(t) \\
P_{6}(t)
\end{array}\right] . }
\end{aligned}
$$

To compute the state probabilities all derivatives of state probabilities become zero, this will enable us to compute steady state availability by equating L.H.S of (3.10) to zero. Now following Wang and Kuo (2000) and Wang et al. (2006).

In the steady state all the derivative equal to zero, thus from (3.10) above we have

$X_{2} P(\infty)=0$.

Thus, Eq. (3.11) above could be written in matrix form as:

$\left[\begin{array}{ccccccc}-2 \alpha & \beta & \beta & 0 & 0 & 0 & 0 \\ \alpha & -(2 \alpha+\beta) & 0 & \beta & \beta & 0 & 0 \\ \alpha & 0 & -(2 \alpha+\beta) & 0 & 0 & \beta & \beta \\ 0 & \alpha & 0 & -\beta & 0 & 0 & 0 \\ 0 & \alpha & 0 & 0 & -\beta & 0 & 0 \\ 0 & 0 & \alpha & 0 & 0 & -\beta & 0 \\ 0 & 0 & \alpha & 0 & 0 & 0 & -\beta\end{array}\right]\left[\begin{array}{c}P_{0}(\infty) \\ P_{1}(\infty) \\ P_{2}(\infty) \\ P_{3}(\infty) \\ P_{4}(\infty) \\ P_{5}(\infty) \\ P_{6}(\infty)\end{array}\right]$

$$
=\left[\begin{array}{l}
0 \\
0 \\
0 \\
0 \\
0 \\
0 \\
0
\end{array}\right]
$$

Using the normalizing condition below, it follows that

$\sum_{i=0}^{6} P_{i}(\infty)=1$

Solving Eqs. (3.11) and (3.12) to obtain $P_{0}(\infty), P_{1}(\infty), P_{2}(\infty)$ we therefore, obtained the explicit expression for steady state availability as follows:

$\operatorname{Av}_{2}(\infty)=\frac{\beta^{2}+2 \alpha \beta}{4 \alpha^{2}+2 \alpha \beta+\beta^{2}}$
Now to evaluate the MTSF for configuration II, following Wang and Kuo (2000) and Wang et al. (2006), the MTSF of a system could be obtained by deleting the rows and column of the absorbing (failure) state and transposing the new matrix $\mathrm{H}_{2}$. The expected time to reach an absorbing state is

$$
\begin{aligned}
& E\left[T_{p(0) \rightarrow p(\text { absorbing })}\right]=P(0)\left(-H_{2}^{-1}\right)\left[\begin{array}{l}
1 \\
1 \\
1
\end{array}\right], \\
& \text { where } H_{2}=\left[\begin{array}{ccc}
-2 \alpha & \alpha & \alpha \\
\beta & -(2 \alpha+\beta) & 0 \\
\beta & 0 & -(2 \alpha+\beta)
\end{array}\right] .
\end{aligned}
$$

Now for the second system, the explicit expression of $\mathrm{MTSF}_{2}$ is given by

$E\left[T_{p(0) \rightarrow p(\text { absorbing })}\right]=\mathrm{MTSF}_{2}=\frac{2 \alpha^{2}+\alpha \beta+4}{8 \alpha}$.

\section{Availability and meantime to system failure of configuration III}

For the analysis of availability case of configuration III, we define $P_{i}(t), i=1,2,3, \ldots, 6$ to be the probability that the system at time $t \geq 0$ is in state $S_{i}$. Also let $P(t)$ be the probability row vector at time $t \geq 0$. The initial condition for this problem is: $P(0)=\left[P_{0}(0), P_{1}(0), P_{2}(0), P_{3}(0)\right.$, $\left.P_{4}(0), P_{5}(0), P_{6}(0)\right]=[1,0,0,0,0,0,0]$.

We obtain the following differential equations:

$$
\begin{aligned}
& P_{0}^{\prime}(t)=-3 \alpha P_{0}(t)+\beta P_{1}(t)+\beta P_{2}(t)+\beta P_{3}(t) \\
& P_{1}^{\prime}(t)=-\beta P_{1}(t)+\alpha P_{0}(t) \\
& P_{2}^{\prime}(t)=-(3 \alpha+\beta) P_{2}(t)+\alpha P_{0}(t)+\beta P_{4}(t)+\beta P_{5}(t)+\beta P_{6}(t) \\
& P_{3}^{\prime}(t)=-\beta P_{3}(t)+\alpha P_{0}(t) \\
& P_{4}^{\prime}(t)=-\beta P_{4}(t)+\alpha P_{2}(t) \\
& P_{5}^{\prime}(t)=-\beta P_{5}(t)+\alpha P_{2}(t) \\
& P_{6}^{\prime}(t)=-\beta P_{6}(t)+\alpha P_{2}(t)
\end{aligned}
$$

Equation (3.16) is written in matrix form as

$\dot{P}=X_{3} P$,

where

$$
X_{3}=\left[\begin{array}{ccccccc}
-3 \alpha & \beta & \beta & \beta & 0 & 0 & 0 \\
\alpha & -\beta & 0 & 0 & 0 & 0 & 0 \\
\alpha & 0 & -(3 \alpha+\beta) & 0 & \beta & \beta & \beta \\
\alpha & 0 & 0 & -\beta & 0 & 0 & 0 \\
0 & 0 & \alpha & 0 & -\beta & 0 & 0 \\
0 & 0 & \alpha & 0 & 0 & -\beta & 0 \\
0 & 0 & \alpha & 0 & 0 & 0 & -\beta
\end{array}\right] .
$$

With the initial conditions 


$$
\begin{aligned}
P(0) & =\left[P_{0}(0), P_{1}(0), P_{2}(0), P_{3}(0), P_{4}(0), P_{5}(0), P_{6}(0)\right] \\
& =[1,0,0,0,0,0,0] .
\end{aligned}
$$

To obtain the steady state probabilities, we equate R.H.S of (3.17) to zero which is

$X_{3} P(\infty)=0$.

Thus, (3.18) can be written in matrix form as follows

$$
\left[\begin{array}{ccccccc}
-3 \alpha & \beta & \beta & \beta & 0 & 0 & 0 \\
\alpha & -\beta & 0 & 0 & 0 & 0 & 0 \\
\alpha & 0 & -(3 \alpha+\beta) & 0 & \beta & \beta & \beta \\
\alpha & 0 & 0 & -\beta & 0 & 0 & 0 \\
0 & 0 & \alpha & 0 & -\beta & 0 & 0 \\
0 & 0 & \alpha & 0 & 0 & -\beta & 0 \\
0 & 0 & \alpha & 0 & 0 & 0 & -\beta
\end{array}\right]\left[\begin{array}{c}
P_{0}(\infty) \\
P_{1}(\infty) \\
P_{2}(\infty) \\
P_{3}(\infty) \\
P_{4}(\infty) \\
P_{5}(\infty) \\
P_{6}(\infty)
\end{array}\right]=\left[\begin{array}{l}
0 \\
0 \\
0 \\
0 \\
0 \\
0 \\
0
\end{array}\right]
$$

Solving (3.18) using normalizing condition below

$\sum_{i=0}^{6} P_{i}(\infty)=1$

We obtained $P_{0}(\infty), P_{2}(\infty)$ therefore, the explicit expression for $\mathrm{Av}_{3}(\infty)$ is given by

$\operatorname{Av}_{3}(\infty)=P_{0}(\infty)+P_{2}(\infty)=\frac{\beta^{2}+\alpha \beta}{3 \alpha^{2}+3 \alpha \beta+\beta^{2}}$

To evaluate MTSF for configuration III, we follow similar argument used in configurations I and II. It is difficult to evaluate the transient solution of configuration III above, following Wang and Kuo (2000) and Wang et al. (2006) we delete the rows and columns of the absorbing states of matrix $\mathrm{T}$ and take the transpose to obtain a new matrix $\mathrm{H}_{3}$.

$E\left[T_{p(0) \rightarrow p(\text { absorbing })}\right]=P(0)\left(-H_{3}^{-1}\right)\left[\begin{array}{l}1 \\ 1 \\ 1\end{array}\right]$,

where $M=\left[\begin{array}{ccc}-3 \alpha & \alpha & \alpha \\ \beta & -\beta & 0 \\ \beta & 0 & -(3 \alpha+\beta)\end{array}\right]$.

We therefore, obtain the explicit expression of $\mathrm{MTSF}_{3}$ as follows

$E\left[T_{p(0) \rightarrow p \text { (absorbing) }}\right]=\operatorname{MTSF}_{3}=\frac{4 \alpha+\beta}{\alpha(9 \alpha+2 \beta)}$.

\section{Availability and meantime to system failure of configuration IV}

For the analysis of availability case of configuration III, we define $P_{i}(t), i=1,2,3, \ldots, 6$ to be the probability that the system at time $t \geq 0$ is in state $S_{i}$. Also let $P(t)$ be the probability row vector at time $t \geq 0$. The initial condition for this problem is: $P(0)=\left[P_{0}(0), P_{1}(0), P_{2}(0), P_{3}(0)\right.$, $\left.P_{4}(0), P_{5}(0), P_{6}(0)\right]=[1,0,0,0,0,0,0]$.

$P_{0}^{\prime}(t)=-2 \alpha P_{0}(t)+\beta P_{1}(t)+\beta P_{2}(t)$

$P_{1}^{\prime}(t)=-(2 \alpha+\beta) P_{1}(t)+\alpha P_{0}(t)+\beta P_{3}(t)+\beta P_{4}(t)$

$P_{2}^{\prime}(t)=-\beta P_{2}(t)+\alpha P_{0}(t)$

$P_{3}^{\prime}(t)=-(2 \alpha+\beta) P_{3}(t)+\alpha P_{1}(t)+\beta P_{5}(t)+\beta P_{6}(t)$

$P_{4}^{\prime}(t)=-\beta P_{4}(t)+\alpha P_{1}(t)$

$P_{5}^{\prime}(t)=-\beta P_{5}(t)+\alpha P_{3}(t)$

$P_{6}^{\prime}(t)=-\beta P_{6}(t)+\alpha P_{3}(t)$

Equation (3.22) can be expressed in the following as

$\dot{P}=X_{4} P$,

where

$X_{4}=\left[\begin{array}{ccccccc}-2 \alpha & \beta & \beta & 0 & 0 & 0 & 0 \\ \alpha & -(2 \alpha+\beta) & 0 & \beta & \beta & 0 & 0 \\ \alpha & 0 & -\beta & 0 & 0 & 0 & 0 \\ 0 & \alpha & 0 & -(2 \alpha+\beta) & 0 & \beta & \beta \\ 0 & \alpha & 0 & 0 & -\beta & 0 & 0 \\ 0 & 0 & 0 & \alpha & 0 & -\beta & 0 \\ 0 & 0 & 0 & \alpha & 0 & 0 & -\beta\end{array}\right]$.

For the availability of configuration IV, we consider the initial conditions as $P(0)=\left[P_{0}(0), P_{1}(0), P_{2}(0), P_{3}(0)\right.$, $\left.P_{4}(0), P_{5}(0), P_{6}(0)\right]=[1,0,0,0,0,0,0]$.

In steady state all derivative of state probabilities become zero, thus setting R.H.S of (3.24) to zero become $X_{4} P(\infty)=0$.

This could be written in matrix form as follows:

$\left[\begin{array}{ccccccc}-2 \alpha & \beta & \beta & 0 & 0 & 0 & 0 \\ \alpha & -(2 \alpha+\beta) & 0 & \beta & \beta & 0 & 0 \\ \alpha & 0 & -\beta & 0 & 0 & 0 & 0 \\ 0 & \alpha & 0 & -(2 \alpha+\beta) & 0 & \beta & \beta \\ 0 & \alpha & 0 & 0 & -\beta & 0 & 0 \\ 0 & 0 & 0 & \alpha & 0 & -\beta & 0 \\ 0 & 0 & 0 & \alpha & 0 & 0 & -\beta\end{array}\right]\left[\begin{array}{c}P_{0}(\infty) \\ P_{1}(\infty) \\ P_{2}(\infty) \\ P_{3}(\infty) \\ P_{4}(\infty) \\ P_{5}(\infty) \\ P_{6}(\infty)\end{array}\right]=\left[\begin{array}{l}0 \\ 0 \\ 0 \\ 0 \\ 0 \\ 0 \\ 0\end{array}\right]$

Solving Eq. (3.25) and using normalizing condition

$\sum_{i=0}^{6} P_{i}(\infty)=1$.

The steady state availability equation is given by

$$
\begin{aligned}
\operatorname{Av}_{4}(\infty) & =P_{0}(\infty)+P_{1}(\infty)+P_{3}(\infty) \\
& =\frac{\alpha^{3}+\alpha \beta^{2}+\alpha^{2} \beta}{2 \alpha^{3}+2 \alpha^{2} \beta+2 \alpha \beta^{2}+\beta^{3}} .
\end{aligned}
$$

To compute the explicit expression of meantime to system failure. We follow similar argument as above. It is difficult to evaluate the transient solution, we apply the same procedure as above, and the expected time to reach an absorbing state can be evaluated from 
$E\left[T_{p(0) \rightarrow p(\text { absorbing })}\right]=P(0)\left(-H_{4}^{-1}\right)\left[\begin{array}{l}1 \\ 1 \\ 1\end{array}\right]$,

where $H_{4}=\left[\begin{array}{ccc}-2 \alpha & \alpha & 0 \\ \beta & -(2 \alpha+\beta) & \beta \\ \beta & 0 & -(2 \alpha+\beta)\end{array}\right]$.

The explicit expression for the $\mathrm{MTSF}_{4}$ is given by

$E\left[T_{p(0) \rightarrow p(\text { absorbing })}\right]=\mathrm{MTSF}_{4}=\frac{7 \alpha^{2}+4 \alpha \beta+\beta^{2}}{\alpha\left(8 \alpha^{2}+4 \alpha \beta+\beta^{2}\right)}$.

\section{Analytical comparison of four configurations}

The main purpose of this section is to present analytical comparisons between the configurations to determine the optimal configuration with respect to steady-state availability and mean time to system failure using MAPLE software.

$$
\begin{aligned}
\operatorname{Av}_{1}(\infty)-\operatorname{Av}_{2}(\infty) & =\frac{\beta(\beta+2 \alpha)^{2} \alpha}{\left(\alpha^{2}+2 \alpha \beta+\beta^{2}\right)\left(3 \alpha^{2}+3 \alpha \beta+\beta^{2}\right)} \\
\forall \alpha, \beta>0 & \Rightarrow \operatorname{Av}_{1}(\infty)>\operatorname{Av}_{2}(\infty)
\end{aligned}
$$

$$
\begin{aligned}
& \operatorname{Av}_{3}(\infty)-\operatorname{Av}_{2}(\infty) \\
& =\frac{\beta(\beta+2 \alpha) \alpha(\alpha-\beta)}{\left(4 \alpha^{2}+2 \alpha \beta+\beta^{2}\right)\left(3 \alpha^{2}+3 \alpha \beta+\beta^{2}\right)} \Rightarrow \operatorname{Av}_{3}(\infty) \\
& >\operatorname{Av}_{2}(\infty), \quad \alpha>\beta \\
& \operatorname{Av}_{1}(\infty)-\operatorname{Av}_{4}(\infty) \\
& \quad=\frac{\beta \alpha\left(3 \alpha^{2} \beta+2 \beta^{2} \alpha+\beta^{3}+3 \alpha^{3}\right)}{\left(\alpha^{2}+2 \alpha \beta+\beta^{2}\right)\left(2 \alpha^{3}+2 \alpha^{2} \beta+2 \beta^{2} \alpha+\beta^{3}\right)} \\
& \Rightarrow \operatorname{Av}_{1}(\infty)>\operatorname{Av}_{4}(\infty), \quad \forall \alpha, \beta>0 \\
& \operatorname{Av}_{2}(\infty)-\operatorname{Av}_{4}(\infty) \\
& \quad=\frac{\beta^{3} \alpha(\alpha-\beta)}{\left(2 \alpha^{3}+2 \alpha^{2} \beta+2 \beta^{2} \alpha+\beta^{3}\right)\left(4 \alpha^{2}+2 \alpha \beta+\beta^{2}\right)} \\
& \Rightarrow \operatorname{Av}_{2}(\infty)>\operatorname{Av}_{4}(\infty), \quad \alpha>\beta \\
& \operatorname{Av}_{1}(\infty)-\operatorname{Av}_{3}(\infty)=\frac{\beta(\beta+2 \alpha)^{2} \alpha}{\left(\alpha^{2}+2 \alpha \beta+\beta^{2}\right)\left(3 \alpha^{2}+3 \alpha \beta+\beta^{2}\right)} \\
& \quad \Rightarrow \operatorname{Av}_{1}(\infty)>\operatorname{Av}_{3}(\infty)
\end{aligned}
$$

$$
\begin{aligned}
& \operatorname{Av}_{3}(\infty)-\operatorname{Av}_{4}(\infty) \\
& \quad=\frac{\beta \alpha^{2}(\alpha-\beta)}{\left(3 \alpha^{2}+3 \alpha \beta+\beta^{2}\right)\left(2 \alpha^{3}+2 \alpha^{2} \beta+2 \beta^{2} \alpha+\beta^{3}\right)} \\
& \quad \Rightarrow \operatorname{Av}_{3}(\infty)>\operatorname{Av}_{4}(\infty), \quad \alpha>\beta
\end{aligned}
$$

$$
\Rightarrow \operatorname{Av}_{1}(\infty)>\operatorname{Av}_{3}(\infty)>\operatorname{Av}_{2}(\infty)>\operatorname{Av}_{4}(\infty)
$$$$
\begin{aligned}
& \mathrm{MTTF}_{1}-\mathrm{MTTF}_{2}=\frac{\beta+2 \alpha}{4 \alpha^{2}} \Rightarrow \mathrm{MTSF}_{1}>\mathrm{MTSF}_{2}, \\
& \forall \alpha, \beta>0
\end{aligned}
$$

$$
\begin{aligned}
\mathrm{MTSF}_{1}-\mathrm{MTSF}_{3} & =\frac{19 \alpha^{2}+13 \alpha \beta+2 \beta^{2}}{2 \alpha^{2}(9 \alpha+2 \beta)} \\
\forall \alpha, \beta>0 & \Rightarrow \mathrm{MTSF}_{1}>\mathrm{MTSF}_{3},
\end{aligned}
$$$$
\mathrm{MTSF}_{1}-\mathrm{MTSF}_{4}=\frac{10 \alpha^{3}+12 \alpha^{2} \beta+7 \beta^{2} \alpha+\beta^{3}}{2 \alpha^{2}\left(8 \alpha^{2}+4 \alpha \beta+\beta^{2}\right)}
$$$$
\Rightarrow \mathrm{MTSF}_{1}>\mathrm{MTSF}_{4} \text {, }
$$

$$
\forall \alpha, \beta>0
$$

$$
\begin{aligned}
\mathrm{MTSF}_{2}-\mathrm{MTSF}_{3} & =\frac{13 \alpha \beta+2 \beta^{2}+20 \alpha^{2}}{4 \alpha^{2}(9 \alpha+2 \beta)} \\
\forall \alpha, \beta>0 & \Rightarrow \mathrm{MTSF}_{2}>\mathrm{MTSF}_{3},
\end{aligned}
$$

$$
\begin{aligned}
& \operatorname{MTSF}_{2}-\mathrm{MTSF}_{4}=\frac{8 \alpha^{2} \beta+8 \beta^{2} \alpha+\beta^{3}+4 \alpha^{3}}{4 \alpha^{2}\left(8 \alpha^{2}+4 \alpha \beta+\beta^{2}\right)} \\
& \quad \Rightarrow \operatorname{MTSF}_{2}>\mathrm{MTSF}_{4} \\
& \forall \alpha, \beta>0 \\
& \Rightarrow \operatorname{MTTF}_{1}(\infty)>\operatorname{MTTF}_{2}(\infty)>\operatorname{MTTF}_{3}(\infty)>\operatorname{MTTF}_{V 4}(\infty)
\end{aligned}
$$

It is evident from Eqs. (4.1) to (4.11) that the optimal configuration is configuration $\mathrm{I}$.

\section{Numericals comparison of four configurations}

In this section, numerical comparison for the results of availability, MTSF, and profit function for all the developed models was presented. For each configuration the following set of parameters values are fixed throughout the simulations for consistency: $\alpha=0.4, \beta=0.3$.

The results which compare the steady state availability and mean time to system failure with respect to $\alpha$ and $\beta$ for all the four systems considered depicted in Figs. 1, 2, 3 and 4. Figures 1 and 4 shows that the steady-state availability and MTSF decrease as $\alpha$ increases for any configuration. Furthermore, configuration I seem to be most effective and reliable configuration among the four configurations. It is clear from Figs. 2 and 3 that the steady state availability and MTSF increase as $\beta$ increases for any configuration. It is evident from Figs. 2 and 3 that configuration $\mathrm{I}$ is more reliable Thus, configuration $\mathrm{I}$ is the optimal configuration in this study.

Numerical simulations of the configurations I, II, III and IV are depicted in Figs. 5-8 using the cost presented in Table 1. 


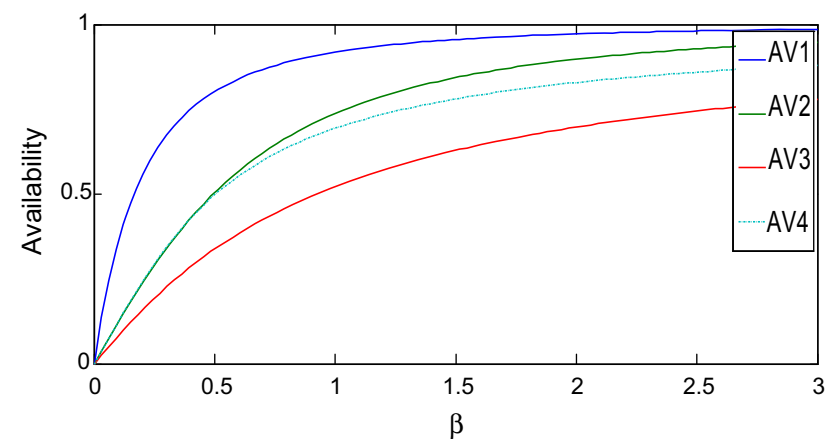

Fig. 2 Availability versus $\beta$

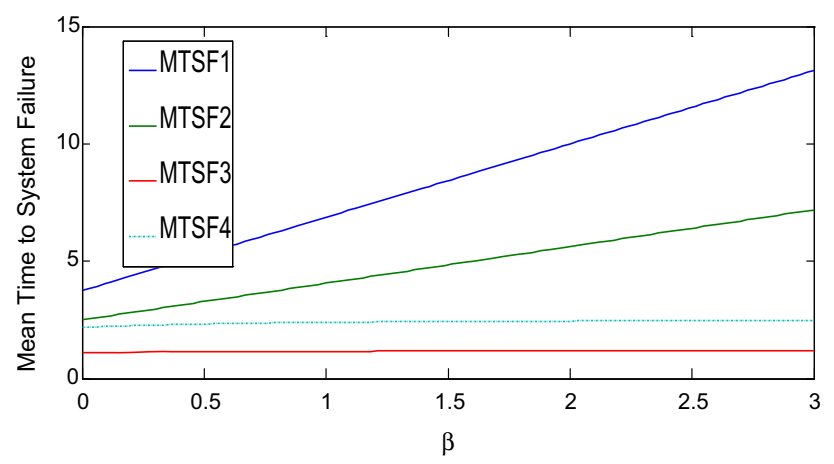

Fig. 3 MTSF versus $\beta$

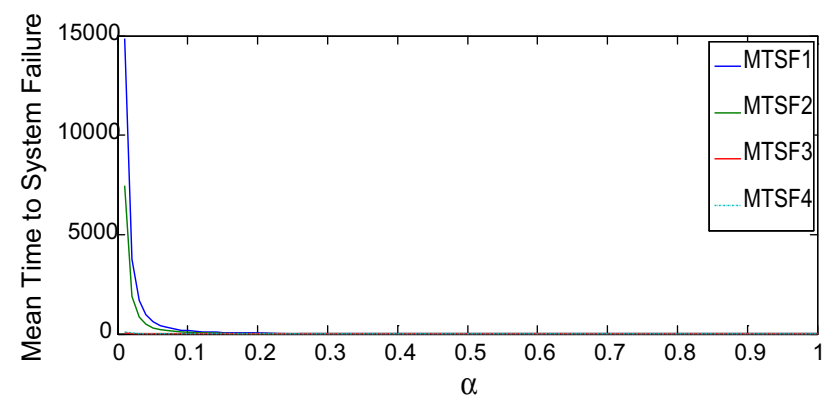

Fig. 4 MTSF versus $\alpha$

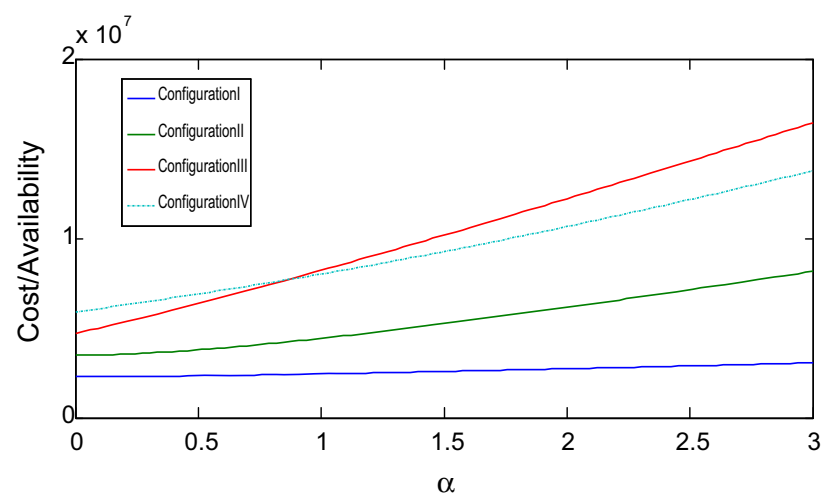

Fig. 5 Cost/availability versus failure rate

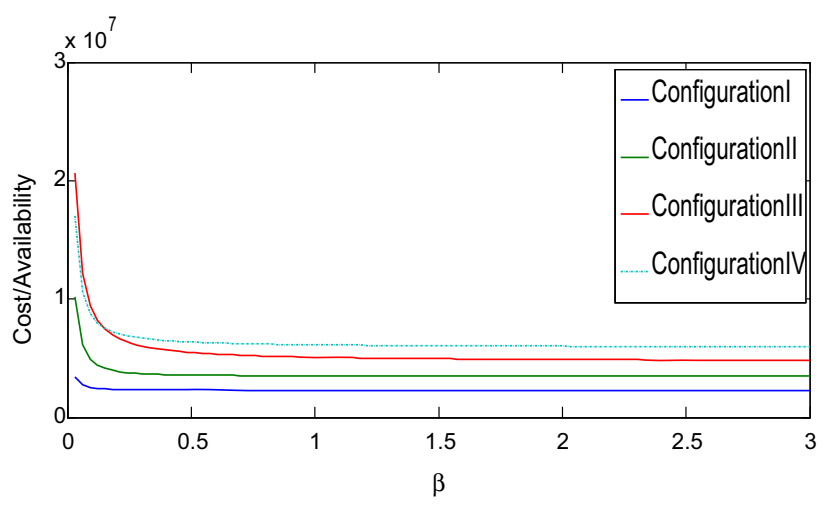

Fig. 6 Cost/MTSF versus repair rate

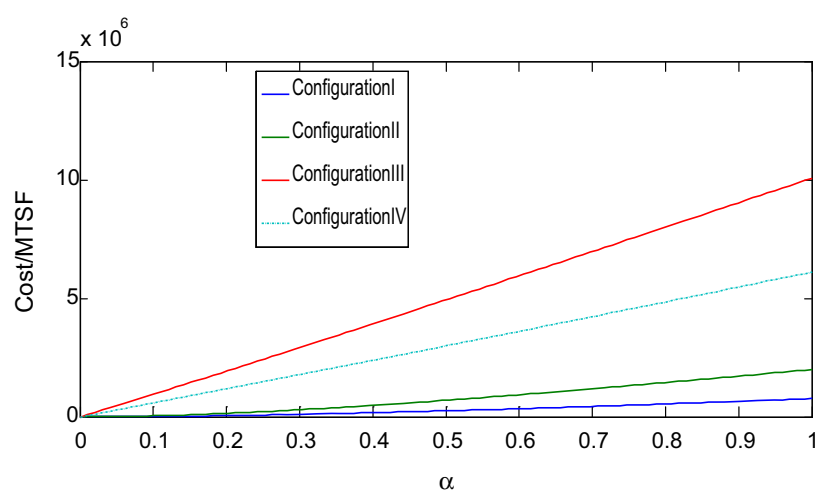

Fig. 7 Cost/MTSF versus failure rate

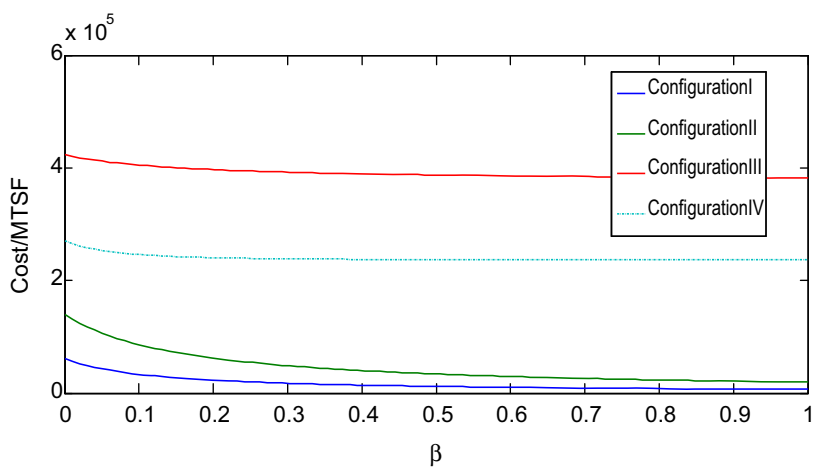

Fig. 8 Cost/MTSF versus repair rate

Table 1 Cost for configurations

\begin{tabular}{ll}
\hline Configuration & Cost \\
\hline Configuration I & $\$ 2,300,000$ \\
Configuration II & $\$ 3,500,000$ \\
Configuration III & $\$ 4,700,000$ \\
Configuration IV & $\$ 5,900,000$ \\
\hline
\end{tabular}

Figures 5 and 7 shows that cost/availability and cost/ MTSF increases as $\alpha$ increases for any configuration. On the other hand, it is clear from Figs. 6 and 8 cost/ 
availability and cost/MTSF decreases as $\beta$ increases for any configuration. It is evident from Figs. 5, 6, 7 and 8, that the optimal configuration is configuration I.

\section{Conclusion}

With the advancement made in the field of modern technology, configuration of photovoltaic systems becomes more and more complex. These complexities reduce the quality and productivity of the photovoltaic systems. Therefore, it is vital to have reliable photovoltaic systems for efficiency, long-term survival and growth. Thus, these systems are expected to remain operative with the maximum efficiency for the maximum duration to ensure their reliable operation. To overcome this problem, redundancy is used in this paper as an effective technique for reliability enhancement of photovoltaic systems. Photovoltaic systems attained high reliability using cold standby redundant units as depicted in this paper. We constructed four different photovoltaic configurations with maximum voltage of 24 each, with cold standby units to study the cost/benefit analysis of four configurations under uncertainty. Explicit expressions for the mean time to system failure and steady state availability have been developed. Comparison was performed using ranking method, both numerical and analytical. Results have shown that system 1 was the best in terms of availability, meantime to system failure and cost benefit ratio. In this paper, the systems were analyzed analytically using MAPLE and numerically using MATLAB and from the result obtained using both software; the optimal configuration is configuration I. The developed models will help in determining the maintenance policy, which will ensure the maximum overall availability and MTSF. The present study will help the reliability analyst, engineers and system designers to develop sophisticated models and to design more critical system in interest of human kind. The study will also assist engineers, decision makers and plant management to avoid an incorrect reliability assessment and consequent erroneous decision making which may lead to unnecessary expenditures, incorrect maintenance scheduling and reduction of safety standards. There are several further research topics which will be studied in the future as follows. First, further work should be done to determine the impact of online and offline preventive maintenance to the system. Second, photovoltaic systems are more common in practice which components with the same failure rate. So modeling of photovoltaic systems system where the failure rate follows different probability distribution like Weibull, Beta, Gamma, Normal distributions, etc., be addressed.
Acknowledgements The authors are grateful to the anonymous reviewers for their constructive comments which have helped to improve the manuscript.

Open Access This article is distributed under the terms of the Creative Commons Attribution 4.0 International License (http://crea tivecommons.org/licenses/by/4.0/), which permits unrestricted use, distribution, and reproduction in any medium, provided you give appropriate credit to the original author(s) and the source, provide a link to the Creative Commons license, and indicate if changes were made.

\section{References}

Cichocki A (2001) Limit reliability of some homogeneous regular series-parallel and parallel-series systems. Appl Math Comput 117(1):55-72

Deepankar S, Jitendra KR, Gupta S (2010) Measurement of reliability parameters for solar (PV) system by employing Boolean function techniques. Res J Math Stat 2(22):81-85

Garg D, Kumar K, Singh J (2010) Availability analysis of a cattle feed plant using matrix method. Int J Eng 3(2):201-219

Juang YS, Lin SS, Kao HP (2008) A knowledge management system for series parallel availability optimization and design. Expert Syst Appl 34(1):181-193

Kołowrocki K (1994) Limit reliability functions of some seriesparallel and parallel-series systems. Appl Math Comput 62(2-3):129-151

Levitin G, Lisnianski A (1999) Joint redundancy and maintenance optimization for multistate series-parallel systems. Reliab Eng Syst Saf 64(1):33-42

Moghaddam TR, Safari J, Sassani F (2008) Reliability optimization of series parallel systems with a choice of redundancy strategies using genetic algorithm. Reliab Eng Syst Saf 93(4):550-556

Moustafa MS (1998) Transient Analysis of Reliability with and without Repair for K-out-of-N: G Systems with M Failure Modes. Reliab Eng Syst Saf 59:317-320

Sarhan AM (2009) Reliability equivalence factors of a general seriesparallel system. Reliab Eng Syst Saf 94(2):229-236

Sarhan AM, Al-Ruzaiza AS, Alwasel IA, El-Gohary AI (2004) Reliability equivalence of a series-parallel system. Appl Math Comput 154(1):257-277

Rao MS, Naikan VNA (2014) Reliability analysis of repairable systems using system dynamics modeling and simulation. J Ind Eng Int 10:69

Sun J, Xi L, Du S, Ju B (2008) Reliability modeling and analysis of serial-parallel hybrid multioperational manufacturing system considering dimensional quality, tool degradation and system configuration. Int J Prod Econ 114:149-164

Wang KH, Kuo CC (2000) Cost and probabilistic analysis of series systems with mixed standby components. Appl Math Model 24:957-967

Wang K, Hsieh C, Liou C (2006) Cost benefit analysis of series systems with cold standby components and a repairable service station. J Qual Technol Quant Manag 3(1):77-92

Yalaoui A, Chu C, Chatelet E (2005) Reliability allocation problem in a series-parallel system. Reliab Eng Syst Saf 90(1):55-61

Yazdanpanah MA (2014) Modeling and sizing optimization of hybrid photovoltaic/wind power generating system. J Ind Eng Int 10(49): $1-4$

Yusuf I (2014) Comparative analysis of profit between three dissimilar repairable redundant systems using supporting external device for operation. J Ind Eng Int 10:77 